\title{
Geochemistry of the extensive peralkaline pyroclastic flow deposit of NW Mexico, based on conventional and handheld X-ray fluorescence. Implications in a regional context
}

\author{
Geoquímica del extenso depósito de flujo piroclástico hiperalcalino del NW de \\ México, basada en fluorescencia de rayos $X$ convencional y portátil. Implicaciones \\ en un contexto regional
}

\author{
J.R. Vidal-Solano ${ }^{1 *}$, R. Lozano Santa Cruz $^{2}$, O. Zamora ${ }^{3}$, A. Mendoza-Cordova ${ }^{1}$, J.M. Stock ${ }^{4}$ \\ I'Área de FRX, Laboratorio de Cristalografía y Geoquímica del Departamento de Geología, Universidad de Sonora, \\ 83000 Hermosillo, Sonora, México \\ ${ }^{2}$ Laboratorio de FRX del Instituto de Geología de la Universidad Nacional Autónoma de México, D.F., México. \\ ${ }^{3}$ Laboratorio de Geoquímica Ambiental del Instituto de Geología de la Universidad Nacional Autónoma de México, \\ D.F., México \\ ${ }^{4}$ California Institute of Technology, Seismo Lab 170-25, 1200 E. California Blvd., Pasadena CA 91125 USA \\ *Corresponding author: jrvidal@ciencias.uson.mx
}

Received: 26/04/2011 / Accepted: 12/04/2013

\begin{abstract}
Chemical analyses conducted on the surface of rock slabs under a combination of two X-ray spectrometry methods, wavelength dispersive XRF and energy dispersive XRF, are used to establish a geochemical correlation between the studied samples. This proves to be an excellent method for the characterization of volcanic glasses, particularly when particles of exotic origin are present, because the effect of these is not easily eliminated by conventional whole rock analysis. Analyses of glassy rhyolites (ignimbrites and lava flows) in northwestern Mexico establish a geochemical signature for the samples, providing criteria that allow us to: a) correlate them with a peralkaline volcanic event, previously reported, that occurred during Middle Miocene time; $b$ ) distinguish them from other metaluminous varieties in the region and, c) propose a correlation between all the peralkaline vitrophyres that crop out within the studied area, of at least $50.000 \mathrm{~km}^{2}$, validating the hypothesis that they are related to the same volcanic event. Finally, based on the results of this study and previous geological investigations, it is proposed that a distance of more than $100 \mathrm{~km}$ between the geographical location of the thickest peralkaline deposits in Sonora, is probably related to a displacement along transtensional dextral faults during the Late Miocene..
\end{abstract}

Keywords: Geochemical correlation, ignimbrites, peralkaline rhyolites, NW México

Resumen

El análisis químico realizado en secciones de roca bajo la combinación de dos métodos de espectrometría de fluorescencia de rayos x, por dispersión de longitudes de onda y por dispersión de energías, permitió establecer una correlación geoquímica entre las muestras estudiadas, resultando ser un excelente método para la caracterización de vidrios volcánicos, en particular cuando contie- 
nen partículas ajenas al magma que no pueden ser eliminadas por el análisis de roca total convencional. Los resultados obtenidos sobre las riolitas vítreas estudiadas (ignimbritas y coladas) del Noroeste de México permiten establecer una firma geoquímica de las muestras que proporciona criterios permitiendo: a) correlacionarlas con un evento volcánico, anteriormente descrito, de tipo hiperalcalino que ocurrió durante el Mioceno medio; b) diferenciarlas de las ignimbritas metaluminosas también presentes en la región y, c) proponer una correlación entre los vitrófiros de la zona estudiada, de al menos $50000 \mathrm{~km} 2$, haciendo válida la hipótesis de que todos ellos provienen de una misma erupción. Finalmente, basados en los resultados de este estudio y de trabajos geológicos previos, se propone que una diferencia de hasta más de $100 \mathrm{~km}$ en la ubicación geográfica de los depósitos hiperalcalinos de mayor espesor, es probablemente relacionada con un desplazamiento a lo largo de fallas dextrales asociadas a un evento extensivo en transtensión ocurrido durante el Mioceno superior.

Palabras clave: Correlación geoquímica, ignimbrites, riolitas hiperalcalinas, NW de México

\section{Introduction}

In Northwestern Mexico, shortly after the Miocene Continental Volcanic Arc became inactive, a Middle Miocene magmatic event occurred, characterized by the eruption of anorogenic melts in a rift environment immediately prior to the Gulf of California opening. This volcanic event comprised some occurrences of mafic lavas with transitional signatures, but was dominated by peralkaline silicic volcanic rocks (Vidal-Solano, 2005). The peralkaline comenditic rocks crop out as lavas and pyroclastic flow deposits. Moreover, an ignimbritic sequence has been widely recognized on both sides of the Gulf of California (Fig. 1), in Baja California where it is known as the Tuff of San Felipe (Stock et al. 1999; Oskin et al. 2001; Bennett, 2009; Olguin-Villa, 2010; OlguinVilla et al, 2010), and in the state of Sonora where it is described as peralkaline ignimbrite (Vidal Solano et al., 2005, 2007, 2008a; Barrera-Guerrero and Vidal-Solano, 2010; Gómez-Valencia and Vidal-Solano, 2010). This ignimbritic episode, whose outcrops span an area of at least $50.000 \mathrm{~km}^{2}$, has been attributed to a possible megaeruption that occurred during Middle Miocene time (Vidal-Solano et al., 2008b). In this paper we test a method of geochemical characterization of the peralkaline pyroclastic deposits recognized in NW Mexico (Vidal-Solano, 2012), with the aim to correlate major and trace element variations in the basal vitreous lithofacies by analyzing flat surfaces of rock slabs.

\section{Methods}

Geochemical methods are useful to correlate outcrops of ignimbrites that belong to the same event in different regions. However, it is important to check first that samples show no alteration and to use elements that have little mobility. Some immobile or slightly mobile trace elements can be determined by X-ray fluorescence (XRF) with great accuracy; this analytical technique is therefore useful to generate good results with low investment of time and resources. The existence of an extensive ignimbrite unit (the Tuff of San Felipe in Baja California and Middle Miocene ignimbrites near Hermosillo), which crops out over an area of at least $50000 \mathrm{Km}^{2}$ in NW Mexico, has been attributed to a possible mega-eruption during Middle Miocene time (Vidal-Solano et al., 2008a). Until now, the correlations between the different outcrops located both in Sonora and Baja California were based on paleomagnetic studies (Oskin et al., 2001; Hernandez-Mendez et al., 2008, Stock et al., 2008), which have shown similar magnetization and an unusual direction for Middle Miocene time (Olguin-Villa et al., 2010). Geochemical analyses of pyroclastic flow deposits may show erratic variations in some elements, due to: 1) the use of whole rock samples that, in the case of ignimbrites, can contain fragments of alien or mixed liquids, and, 2) the poor representativeness of the samples with the required chemical data, because the cost of these analytical techniques restricts the number of analyses (Vidal-Solano and Meza-Figueroa, 2009).

In this work we have used both WD-XRF and ED-XRF techniques to obtain the geochemical data on the surface of rock chips remaining after preparation of thin sections. The analytical method is carried out in a specific point of small size and well located on the surface rock. This method is highly useful, because it allows us to determine original compositions of magmas by analyses of the rock surface which is free from exotic fragments. In addition, such a method represents a very low cost investment in sample collection and preparation for the analysis, because the hand specimen can be re-used and only requires minimal preparation. Furthermore, the time needed on the analytical equipment is minimal; the procedure is very efficient and yields results that are useful complements to the initial petrographic study.

\section{Analytical Techniques}

Analyses reported in this study were carried out at the $\mathrm{X}$-ray Fluorescence Laboratory and the Environmental 
Geochemistry Laboratory of the Universidad Nacional Autónoma de México, using a Siemens SRS 3000 WDXRF sequential spectrometer and a Thermo Scientific portable analyzer ED-XRF Niton XL3T. The SRS 3000 spectrometer was used to measure the trace elements $\mathrm{Rb}, \mathrm{Sr}, \mathrm{Ba}, \mathrm{Y}, \mathrm{Zr}, \mathrm{Nb}, \mathrm{V}, \mathrm{Cr}, \mathrm{Co}, \mathrm{Ni}, \mathrm{Cu}, \mathrm{Zn}$, Th and $\mathrm{Pb}$, under the analytical and reference materials used in the construction of calibration curves reported in Lozano and Bernal (2005). To shorten the total measurement time, an adjustment was made spending only 20 seconds per point, reducing therefore the time per sample to ca. $15 \mathrm{~min}$. With the Niton XL3T spectrometer we measured Rb, Sr, Zr, Zn and $\mathrm{Pb}$, but also some major elements like $\mathrm{Mn}, \mathrm{Ti}, \mathrm{Fe}, \mathrm{Ca}$ and $\mathrm{K}$ on three different areas of one square centimeter on each rock slab. All these values were corrected using standard analysis of the IGL series (IGLSY, IGLA-1 and IGLS-1) and RGM-1. The total measurement time per sample was 3 minutes, and the variables of the analysis are reported in Zamora et al. (2008). IGLA-1 and RGM-1 were analyzed by both techniques under the same conditions in order to assure that analytical results were well calibrated. Trace elements measured with the SRS3000 showed an excellent agreement between measured and certified values with a mean accuracy of $93.4 \%$. Values for IGLA-1 reported by Niton showed for $\mathrm{Fe}, \mathrm{Ca}$ and $\mathrm{K}$ a mean accuracy of $88 \%$ calculated by $A=100-A b s[($ True value-Meas value)/Meas value)*100)].

\section{Sample preparation}

The sample preparation consists in smoothing, cleaning and drying the surface of each slab to expose the flat side to the $\mathrm{x}$-ray. This is not difficult because after the preparation of thin sections the slabs already had a flat surface, as well as a suitable size to adjust them on the sample loader of the SRS 3000 spectrometer (Fig. 2). Samples smaller than $34 \mathrm{~mm}$ were mounted on a plastic film inert to Xrays (mylar) prior to the analysis.
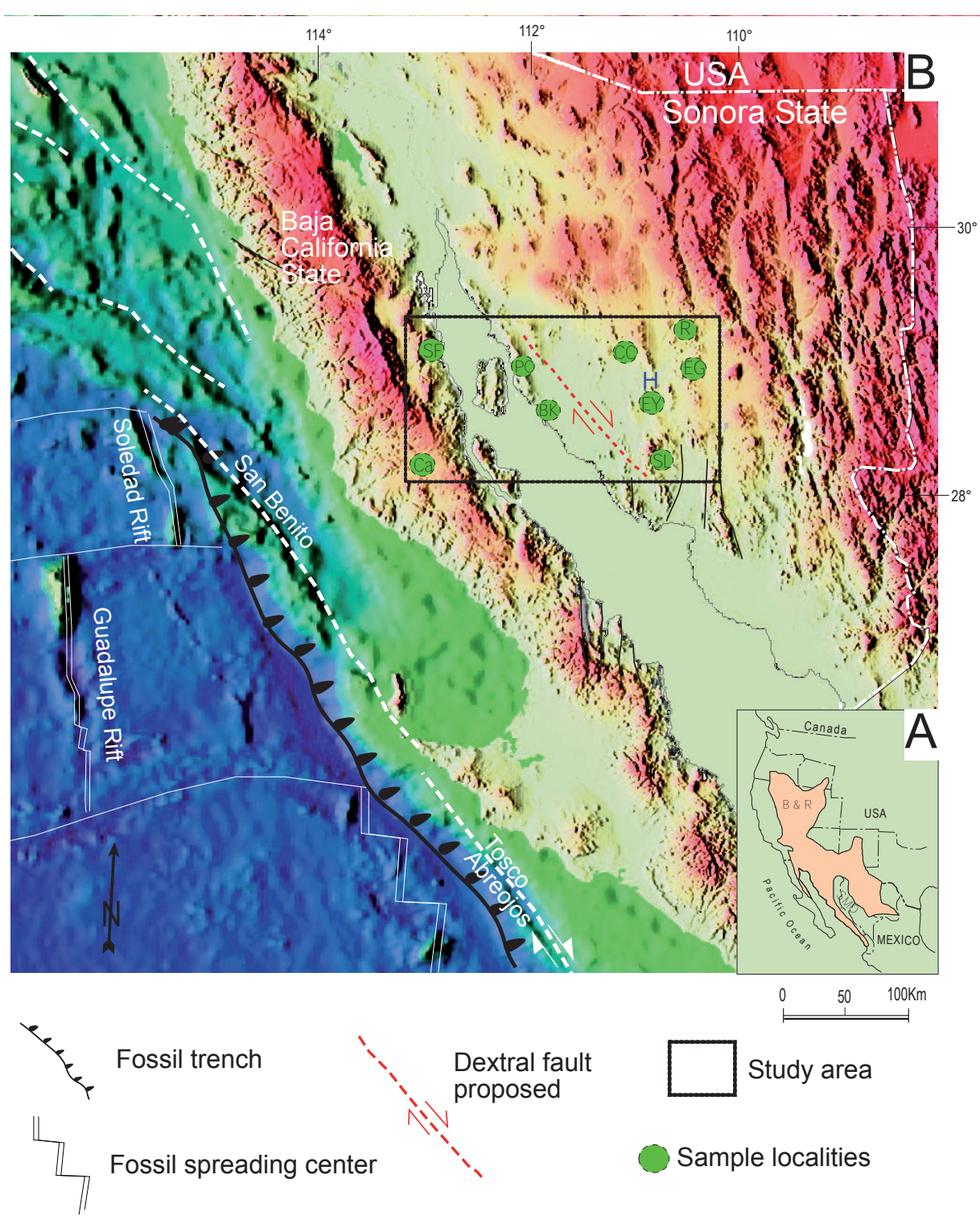

Fig. 1.- A: Geographical location of the NW of Mexico showing the province of the Sierra Madre Occidental (SMO) and the areas affected by Miocene extensional tectonics (Basin and Range). B: Tectonic reconstruction for Middle Miocene time (12 Ma) showing the initial position of the northern part of the Baja California peninsula relative to Sonora, modified from Vidal-Solano et al. (2008a). Location of the proposed system of dextral faults that displace sites of Kino Bay and Punta Chueca is illustrated. SF, San Felipe; Ca, Cataviña; PC, Punta Chueca; BK, Bahía de Kino; SL, Sierra Libre; CC, Cerro La Ceja; H, Hermosillo; EY, Cerro El Yeso; R, Rayón; EG, El Gavilán.

Fig. 1.- A: Localización geográfica del NW de México mostrando la provincia de la Sierra Madre Occidental (SMO) y las zonas afectadas por la tectónica extensional del Mioceno (Basin and Range). B: Reconstrucción tectónica para el Mioceno medio (12 Ma) de la posición inicial de la parte norte de la Península de Baja California con respecto a Sonora, modificado de Vidal-Solano et al. (2008a). Se representa la ubicación del sistema propuesto de fallas dextrales que desplazan los sitios de Bahía Kino y Punta Chueca. SF, San Felipe; Ca, Cataviña; PC, Punta Chueca; BK, Bahía de Kino; SL, Sierra Libre; CC, Cerro La Ceja; H, Hermosillo; EY, Cerro El Yeso; R, Rayón; EG, El Gavilán. 


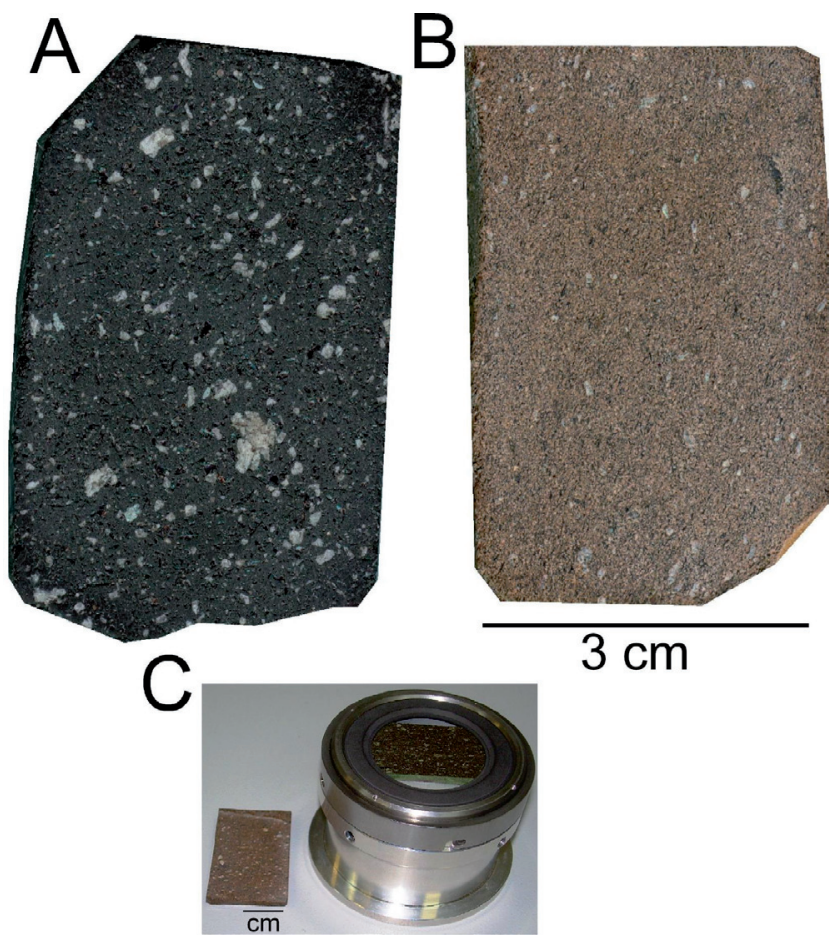

Fig. 2.-Example of rock slabs studied. A: black eutaxitic vitrophyre with porphyritic texture (5\% alkali feldspar), B: brown vitroclastic, almost aphanitic, vitrophyre. C: sample loader used for WD-XRF spectrometer.

Fig. 2.-Ejemplo de las secciones de roca estudiadas, A: vitrófiro negro eutaxítico porfírico de Feldespato alcalino (5\%), B: vitrófiro café vitroclástico casi afanítico. C: porta-muestra utilizado para el análisis por WD-FRX en el espectrómetro WD-FRX.

\section{Studied material}

We studied 35 slabs of peralkaline rocks: 22 samples of vitrophyre facies of the ignimbritic deposits and 13 samples of glassy facies of porphyritic rhyolitic lava flows having the same chemical affinity. One sample of a metaluminous rhyolite was also analyzed for reference. The ignimbritic samples are slightly porphyritic $(<15 \%$ phenocrysts), with different degrees of welding yielding vitroclastic to eutaxitic textures (Fig. 2). Their mineralogical association is characterized by the presence, in order of abundance, of Na-sanidine, greenish iron-rich ferrohedenbergite and fayalite. Plagioclase and hydrous minerals such as biotite or amphibole are never present in these lavas. This distinctive mineral association characterizes comendite-type high-silica rhyolites (Vidal-Solano et al., 2007). Chemically, these rocks have, unlike the metaluminous rhyolites, high $\mathrm{SiO}_{2}(>72 \%)$ and alkali values (7-9 \%), but low alumina $(<12 \%)$ giving rise to their peralkaline affinity, and relatively high iron contents (Table 1, Vidal-Solano et al., 2005, 2007, 2008b). Another important feature is their high concentration in $\mathrm{Rb}$ and $\mathrm{Zr}$, and very low values in $\mathrm{Sr}$ and $\mathrm{Ba}$.

\section{Results}

The peralkaline rhyolitic lavas and the metaluminous rhyolite were used as references for the geochemical discrimination. The results are reported in Tables 1 and 2. $\mathrm{Zr}, \mathrm{Sr}, \mathrm{Rb}, \mathrm{Pb}, \mathrm{Zn}, \mathrm{Ba}, \mathrm{Nb}$, Th, determined by WD-XRF sequential spectrometry, and $\mathrm{Zr}, \mathrm{Sr}, \mathrm{Rb}, \mathrm{Pb}, \mathrm{Zn}, \mathrm{Mn}, \mathrm{Ti}$, $\mathrm{Ba}, \mathrm{Fe}_{2} \mathrm{O}_{3} \mathrm{t}, \mathrm{CaO}$ and $\mathrm{K}_{2} \mathrm{O}$, determined by ED-XRF spectrometry, show concentrations and ratios consistent with the values obtained by ICP-MS analyses on whole rock samples (Vidal-Solano et al., 2005, 2007, 2008b, OlguinVilla, 2010). The concentrations of these elements were reported on binary, ternary and multi-element diagrams to better visualize the relationships and variations among the different samples (Fig. 3 and 4).

All the samples of the peralkaline rhyolite (ignimbrites and lavas) retain similar element ratios (Fig. 3A): low $\mathrm{Sr}$ and $\mathrm{Ba}$, high $\mathrm{Rb}$ and $\mathrm{Th}$, and moderate concentrations in $\mathrm{Nb}$ and $\mathrm{Zr}$ compared to the metaluminous rhyolite. Parallelism of the spectra in this diagram indicates a genetic link for all the peralkaline samples. However, a difference does exist between the concentrations in the ignimbrites and those in the lavas. This is best illustrated on the diagram of Figure 3B, which shows the clustering of the ignimbrite samples, suggesting a good correlation between ignimbrite outcrops, and a wide dispersion of the points representing the rhyolite lava flows. The ratios between $\mathrm{Rb}, \mathrm{K}_{2} \mathrm{O}, \mathrm{Sr}$, and $\mathrm{Fe}_{2} \mathrm{O}_{3}$ concentrations for each ignimbrite sample are plotted in Figure 4A, which allowed the distinction of different data sets. Three groups with similar chemical signature can be defined, suggesting a common source for samples from different outcrops; this in turn leads to a more precise correlation of the different units.

\subsection{Geochemistry}

The major element analyses show that the ignimbrite samples have high but variable concentrations in $\mathrm{K}_{2} \mathrm{O}(2$ to $5 \%$ ) and low $\mathrm{CaO}$ contents $(<1 \%)$. Total $\mathrm{Fe}_{2} \mathrm{O}_{3}$ values are generally higher than $1 \%$ and increase when $\mathrm{Mn}$ and Ti increase. This is also a criterion that differentiates peralkaline rhyolites from metaluminous ones (VidalSolano, 2005).

The trace element characteristics are best visualized on a multi-element diagram (Fig. 3). Incompatible multi-element patterns, normalized to N-MORB (Pearce, 1983), of the peralkaline ignimbrites exhibit an overall parallelism of the spectra (Fig. 3A), with irregular patterns character- 


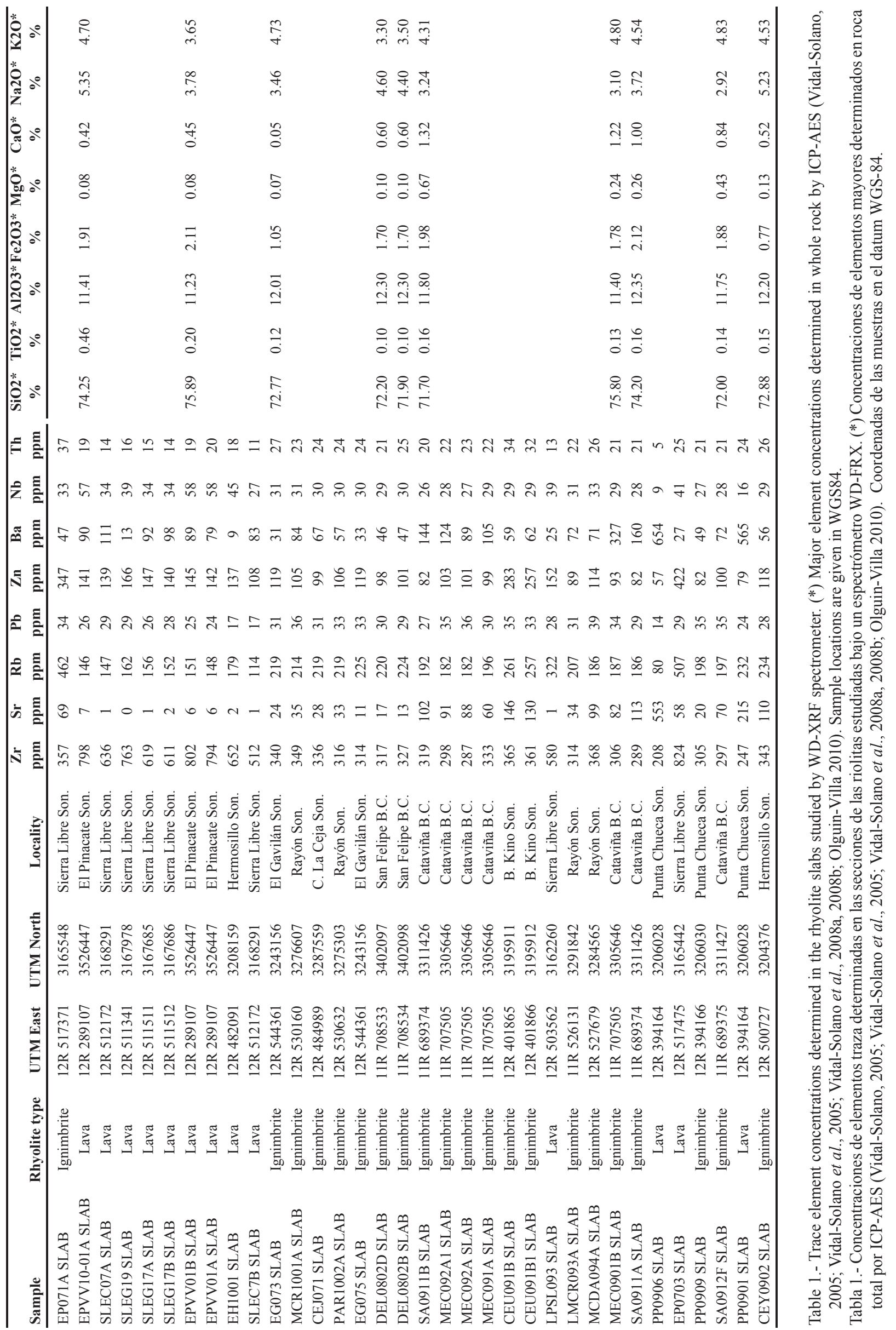




\begin{tabular}{|c|c|c|c|c|c|c|c|c|c|c|c|c|c|c|c|}
\hline Sample & Rhyolite type & UTM East & UTM North & Locality & $\begin{array}{c}\mathrm{Zr} \\
\mathrm{ppm}\end{array}$ & $\begin{array}{c}\mathrm{Sr} \\
\mathbf{p p m}\end{array}$ & $\begin{array}{c}\mathbf{R b} \\
\mathbf{p p m}\end{array}$ & $\begin{array}{c}\text { Pb } \\
\text { ppm }\end{array}$ & $\begin{array}{c}\mathrm{Zn} \\
\mathrm{ppm}\end{array}$ & $\begin{array}{l}\text { Mn } \\
\text { ppm }\end{array}$ & $\begin{array}{c}\text { Ti } \\
\text { ppm }\end{array}$ & $\begin{array}{c}\text { Ba } \\
\text { ppm }\end{array}$ & $\begin{array}{c}\mathrm{Fe} 203 \\
\% \\
\end{array}$ & $\begin{array}{c}\mathrm{CaO} \\
\% \\
\end{array}$ & $\begin{array}{c}\mathrm{K} 2 \mathrm{O} \\
\%\end{array}$ \\
\hline DEL-08-02 D-1 & Ignimbrite & 11R 708533 & 3402097 & San Felipe B.C. & 265 & 17 & 142 & 23 & 53 & 191 & 102 & $<$ LOD & 0.92 & 0.38 & 3.44 \\
\hline DEL-08-02 D-2 & Ignimbrite & 11R 708534 & 3402098 & San Felipe B.C. & 272 & 18 & 145 & 29 & 61 & 230 & 48 & $<$ LOD & 1.03 & 0.45 & 3.51 \\
\hline DEL-08-02 D-3 & Ignimbrite & 11R 708535 & 3402099 & San Felipe B.C. & 200 & 24 & 112 & 19 & 51 & 147 & 124 & $<$ LOD & 0.82 & 0.42 & 3.52 \\
\hline SA 0911 B-1 & Ignimbrite & 11R 692643 & 3312349 & Cataviña B.C. & 255 & 71 & 137 & 28 & 52 & 210 & 527 & $<$ LOD & 1.17 & 1.18 & 4.34 \\
\hline SA 0911 B-2 & Ignimbrite & 11R 692644 & 3312350 & Cataviña B.C. & 319 & 75 & 125 & 31 & 55 & 216 & 564 & $<$ LOD & 1.1 & 1.09 & 4.17 \\
\hline SA 0911 B-3 & Ignimbrite & 11R 692645 & 3312351 & Cataviña B.C. & 265 & 84 & 133 & 24 & 64 & 173 & 600 & $<$ LOD & 1.09 & 1.08 & 4.34 \\
\hline eg $07-5-1$ & Ignimbrite & 12R 544359 & 3243154 & El Gavilán Son. & 325 & 10 & 147 & 20 & 71 & 222 & 847 & $<\mathrm{LOD}$ & 1.1 & 0.32 & 5.32 \\
\hline eg $07-5-2$ & Ignimbrite & 12R 544360 & 3243155 & El Gavilán Son. & 259 & 14 & 142 & 30 & 79 & 182 & 378 & $<$ LOD & 1.12 & 0.39 & 5.13 \\
\hline eg $07-5-3$ & Ignimbrite & 12R 544361 & 3243156 & El Gavilán Son. & 264 & 10 & 146 & 25 & 68 & 215 & 241 & $<$ LOD & 0.98 & 0.33 & 5.34 \\
\hline mec 0901 b-1 & Ignimbrite & 11R 707505 & 3305646 & Cataviña B.C. & 262 & 84 & 131 & 24 & 67 & 308 & 345 & 669 & 1.02 & 1.24 & 4.43 \\
\hline mec $0901 \mathrm{~b}-2$ & Ignimbrite & 11R 707506 & 3305647 & Cataviña B.C. & 253 & 94 & 126 & 24 & 79 & 460 & 288 & 558 & 1.04 & 0.89 & 4.36 \\
\hline mec 0901 b-3 & Ignimbrite & 11R 707507 & 3305648 & Cataviña B.C. & 254 & 52 & 126 & 23 & 68 & 167 & 403 & $<\mathrm{LOD}$ & 1.01 & 1.5 & 4.5 \\
\hline mlcr09-03a-1 & Ignimbrite & 11R 526131 & 3291842 & Rayón Son. & 265 & 26 & 146 & 26 & 65 & 257 & 287 & $<$ LOD & 1.06 & 0.39 & 5.22 \\
\hline mlcr09-03a-2 & Ignimbrite & 11R 526132 & 3291843 & Rayón Son. & 264 & 35 & 146 & 23 & 49 & 257 & 335 & $<$ LOD & 1.09 & 0.4 & 4.83 \\
\hline mlcr09-03a-3 & Ignimbrite & 11R 526133 & 3291844 & Rayón Son. & 273 & 23 & 144 & 21 & 46 & 190 & 244 & $<$ LOD & 1.03 & 0.4 & 4.92 \\
\hline ceu 09b1-1 & Ignimbrite & 12R 401865 & 3195911 & B. Kino Son. & 283 & 101 & 140 & 27 & 150 & 184 & 222 & $<$ LOD & 1.01 & 0.73 & 2.25 \\
\hline ceu $09 \mathrm{~b} 1-2$ & Ignimbrite & 12R 401866 & 3195912 & B. Kino Son. & 270 & 98 & 135 & 28 & 136 & 223 & 360 & $<$ LOD & 1.01 & 0.74 & 2.4 \\
\hline ceu $09 \mathrm{~b} 1-3$ & Ignimbrite & 12R 401867 & 3195913 & B. Kino Son. & 247 & 96 & 131 & 25 & 150 & 171 & 461 & $<$ LOD & 1.08 & 0.8 & 2.45 \\
\hline pp09-06-1 & Lava & 12R 394164 & 3206028 & Punta Chueca Son. & 139 & 457 & 58 & 11 & 48 & 416 & 3261 & 903 & 2.19 & 1.99 & 2.17 \\
\hline pp09-06-2 & Lava & 12R 394165 & 3206029 & Punta Chueca Son. & 162 & 452 & 55 & 12 & 54 & 335 & 3301 & 927 & 2.12 & 1.93 & 2.06 \\
\hline pp09-06-3 & Lava & 12R 394166 & 3206030 & Punta Chueca Son. & 151 & 510 & 58 & 11 & 37 & 403 & 3145 & 1221 & 2.13 & 2.2 & 2.32 \\
\hline ep 07-03-1 & Lava & 12R 517475 & 3165442 & Sierra Libre Son. & 630 & 13 & 238 & 22 & 198 & 280 & 623 & $<$ LOD & 1.44 & 0.27 & 2.15 \\
\hline ep $07-03-2$ & Lava & 12R 517476 & 3165443 & Sierra Libre Son. & 629 & 52 & 217 & 22 & 230 & 171 & 684 & $<$ LOD & 1.34 & 0.69 & 2 \\
\hline ep 07-03-3 & Lava & 12R 517477 & 3165444 & Sierra Libre Son. & 633 & 19 & 230 & 21 & 211 & 282 & 695 & $<$ LOD & 1.37 & 0.31 & 2.06 \\
\hline par10 $02 \mathrm{a}-1$ & Ignimbrite & 12R 530632 & 3275303 & Rayón Son. & 281 & 22 & 156 & 28 & 59 & 195 & 668 & $<$ LOD & 1.15 & 0.36 & 5.06 \\
\hline par10 $02 \mathrm{a}-2$ & Ignimbrite & 12R 530633 & 3275304 & Rayón Son. & 360 & 19 & 148 & 23 & 77 & 214 & 523 & $<$ LOD & 1.1 & 0.34 & 4.88 \\
\hline par10 $02 \mathrm{a}-3$ & Ignimbrite & 12R 530634 & 3275305 & Rayón Son. & 285 & 20 & 156 & 18 & 59 & 162 & 158 & $<\mathrm{LOD}$ & 1.03 & 0.36 & 5.04 \\
\hline cej 07-1-1 & Ignimbrite & 12R 484989 & 3287559 & C. La Ceja Son. & 277 & 14 & 153 & 28 & 68 & 188 & 426 & $<$ LOD & 1.11 & 0.37 & 4.75 \\
\hline cej $07-1-2$ & Ignimbrite & 12R 484990 & 3287560 & C. La Ceja Son. & 270 & 30 & 150 & 21 & 54 & 249 & 423 & $<$ LOD & 1.08 & 0.39 & 4.67 \\
\hline cej 07-1-3 & Ignimbrite & 12R 484991 & 3287561 & C. La Ceja Son. & 260 & 19 & 152 & 28 & 82 & 159 & 307 & $<$ LOD & 1.05 & 0.37 & 4.87 \\
\hline eg $07-3-1$ & Ignimbrite & 12R 544359 & 3243154 & El Gavilán Son. & 268 & 17 & 144 & 27 & 69 & 186 & 248 & $<$ LOD & 1.08 & 0.39 & 4.92 \\
\hline eg $07-3-2$ & Ignimbrite & 12R 544360 & 3243155 & El Gavilán Son. & 274 & 16 & 146 & 21 & 80 & 188 & 636 & $<$ LOD & 1.1 & 0.37 & 5.3 \\
\hline eg $07-3-3$ & Ignimbrite & 12R 544361 & 3243156 & El Gavilán Son. & 275 & 20 & 143 & 25 & 83 & 168 & 271 & $<$ LOD & 1.02 & 0.41 & 5.08 \\
\hline mcr10-01a-1 & Ignimbrite & 12R 530160 & 3276607 & Rayón Son. & 271 & 28 & 151 & 19 & 56 & 153 & 288 & $<$ LOD & 1.1 & 0.28 & 5.16 \\
\hline mcr10-01a-2 & Ignimbrite & 12R 530161 & 3276608 & Rayón Son. & 390 & 27 & 147 & 25 & 64 & 202 & 509 & $<$ LOD & 1.15 & 0.32 & 5.32 \\
\hline mcr10-01a-3 & Ignimbrite & 12R 530162 & 3276609 & Rayón Son. & 284 & 29 & 145 & 33 & 68 & 160 & 247 & $<$ LOD & 1.13 & 0.4 & 5.14 \\
\hline mec 09-01 a-1 & Ignimbrite & 11R 707505 & 3305646 & Cataviña B.C. & 234 & 47 & 129 & 29 & 59 & 245 & 305 & $<$ LOD & 1.03 & 0.55 & 4.5 \\
\hline mec 09-01 a-2 & Ignimbrite & 11R 707506 & 3305647 & Cataviña B.C. & 253 & 45 & 138 & 24 & 66 & 207 & 255 & $<$ LOD & 1.06 & 0.52 & 4.56 \\
\hline mec $09-01$ a-3 & Ignimbrite & 11R 707507 & 3305648 & Cataviña B.C. & 248 & 50 & 124 & 22 & 60 & 142 & 447 & $<$ LOD & 1.04 & 0.57 & 4.6 \\
\hline ceu 09-01b-1 & Ignimbrite & 12R 401865 & 3195911 & B. Kino Son. & 273 & 123 & 132 & 28 & 174 & 123 & 532 & $<$ LOD & 1.03 & 0.74 & 2.37 \\
\hline ceu 09-01b-2 & Ignimbrite & 12R 401865 & 3195911 & B. Kino Son. & 256 & 98 & 136 & 28 & 166 & 281 & 407 & $<\mathrm{LOD}$ & 1.06 & 0.76 & 2.28 \\
\hline ceu $09-01 b-3$ & Ignimbrite & 12R 401866 & 3195912 & B. Kino Son. & 266 & 120 & 134 & 24 & 146 & 183 & 488 & $<$ LOD & 1.05 & 0.79 & 2.51 \\
\hline mcda 09-04a-1 & Ignimbrite & 12R 527679 & 3284565 & Rayón Son. & 299 & 82 & 121 & 34 & 82 & 226 & 260 & $<$ LOD & 1.2 & 0.86 & 3.8 \\
\hline mcda 09-04a-2 & Ignimbrite & 12R 527680 & 3284566 & Rayón Son. & 291 & 76 & 123 & 35 & 74 & 312 & 448 & $<$ LOD & 1.2 & 0.88 & 3.59 \\
\hline mcda 09-04a-3 & Ignimbrite & 12R 527681 & 3284567 & Rayón Son. & 286 & 66 & 125 & 28 & 81 & 242 & 457 & $<$ LOD & 1.26 & 0.77 & 3.65 \\
\hline sa 0912f-1 & Ignimbrite & 11R 689374 & 3311426 & Cataviña B.C. & 271 & 56 & 139 & 25 & 71 & 295 & 390 & $<\mathrm{LOD}$ & 1.27 & 0.6 & 4.62 \\
\hline sa $0912 \mathrm{f}-2$ & Ignimbrite & 11R 689375 & 3311427 & Cataviña B.C. & 288 & 56 & 143 & 29 & 78 & 237 & 481 & $<$ LOD & 1.23 & 0.6 & 4.68 \\
\hline sa 0912f-3 & Ignimbrite & 11R 689376 & 3311428 & Cataviña B.C. & 307 & 59 & 142 & 29 & 68 & 167 & 397 & $<$ LOD & 1.26 & 0.69 & 4.53 \\
\hline $\operatorname{mec} 0902 \mathrm{a} 1-1$ & Ignimbrite & 11R 707505 & 3305646 & Cataviña B.C. & 245 & 71 & 132 & 30 & 63 & 235 & 384 & $<$ LOD & 1.12 & 0.77 & 4.31 \\
\hline $\operatorname{mec} 0902 \mathrm{a} 1-2$ & Ignimbrite & 11R 707506 & 3305647 & Cataviña B.C. & 250 & 68 & 131 & 28 & 62 & 303 & 243 & $<\mathrm{LOD}$ & 1.13 & 0.92 & 4.12 \\
\hline $\operatorname{mec} 0902$ al-3 & Ignimbrite & 11R 707507 & 3305648 & Cataviña B.C. & 389 & 84 & 124 & 26 & 76 & 298 & 829 & $<\mathrm{LOD}$ & 1.26 & 1.03 & 4.38 \\
\hline sa 09-11a-1 & Ignimbrite & 11R 689374 & 3311426 & Cataviña B.C. & 240 & 70 & 130 & 22 & 57 & 238 & 417 & $<$ LOD & 1.14 & 1.23 & 3.99 \\
\hline sa $09-11 \mathrm{a}-2$ & Ignimbrite & 11R 689375 & 3311427 & Cataviña B.C. & 239 & 79 & 130 & 18 & 50 & 270 & 640 & $<\mathrm{LOD}$ & 1.12 & 1.13 & 4.15 \\
\hline sa $09-11 a-3$ & Ignimbrite & 11R 689376 & 3311428 & Cataviña B.C. & 245 & 72 & 128 & 19 & 56 & 173 & 417 & $<$ LOD & 1.11 & 1.07 & 4 \\
\hline cey $09-02-1$ & Ignimbrite & 12R 500727 & 3204376 & Hermosillo. Son. & 291 & 53 & 156 & 26 & 73 & 169 & 185 & $<$ LOD & 1.01 & 0.42 & 4.25 \\
\hline cey $09-02-3$ & Ignimbrite & 12R 500728 & 3204377 & Hermosillo. Son. & 261 & 37 & 159 & 35 & 65 & 234 & 299 & $<$ LOD & 1.04 & 0.41 & 4.24 \\
\hline por $08-02 u-1$ & Ignimbrite & 11R 709412 & 3308830 & Cataviña B.C. & 275 & 29 & 144 & 18 & 52 & 144 & 442 & 322 & 0.99 & 0.43 & 4.38 \\
\hline por $08-02 u-2$ & Ignimbrite & 11R 709413 & 3308831 & Cataviña B.C. & 254 & 39 & 142 & 17 & 70 & 268 & 1609 & 387 & 1.97 & 0.48 & 4.39 \\
\hline por $08-02 u-3$ & Ignimbrite & 11R 709414 & 3308832 & Cataviña B.C. & 296 & 35 & 140 & 20 & 61 & 237 & 710 & 362 & 1.17 & 0.44 & 4.58 \\
\hline pp09-09-1 & Ignimbrite & 12R 394164 & 3206028 & Punta Chueca Son. & 244 & 19 & 143 & 34 & 59 & 199 & 229 & 402 & 0.99 & 1.26 & 4.85 \\
\hline pp09-09-2 & Ignimbrite & 12R 394165 & 3206029 & Punta Chueca Son. & 304 & 12 & 151 & 32 & 58 & 275 & 326 & 410 & 1.05 & 0.62 & 4.49 \\
\hline pp09-09-3 & Ignimbrite & 12R 394166 & 3206030 & Punta Chueca Son. & 252 & 12 & 148 & 32 & 65 & 175 & 342 & 369 & 0.98 & 0.79 & 4.84 \\
\hline pp 09-01-1 & Lava & 12R 394164 & 3206028 & Punta Chueca Son. & 210 & 118 & 183 & 30 & 56 & 175 & 1185 & 642 & 1.08 & 0.64 & 3.13 \\
\hline pp 09-01-2 & Lava & 12R 394165 & 3206029 & Punta Chueca Son. & 271 & 180 & 162 & 30 & 67 & 210 & 2764 & 672 & 1.35 & 1.05 & 2.8 \\
\hline pp 09-01-3 & Lava & 12R 394166 & 3206030 & Punta Chueca Son. & 180 & 96 & 172 & 27 & 53 & 154 & 2189 & 550 & 1.03 & 0.66 & 2.97 \\
\hline del 08-02 b-1 & Ignimbrite & 11R 708533 & 3402097 & San Felipe B.C. & 259 & 10 & 147 & 24 & 68 & 178 & 357 & 367 & 1.09 & 0.34 & 3.51 \\
\hline del 08-02 b-2 & Ignimbrite & 11R 708534 & 3402098 & San Felipe B.C. & 282 & 12 & 150 & 31 & 57 & 254 & 253 & 372 & 1 & 0.35 & 3.76 \\
\hline del 08-02 b-3 & Ignimbrite & 11R 708535 & 3402099 & San Felipe B.C. & 258 & 12 & 149 & 28 & 64 & 235 & 266 & 376 & 0.99 & 0.34 & 3.52 \\
\hline mec 09-02 a-1 & Ignimbrite & 11R 707505 & 3305646 & Cataviña B.C. & 204 & 71 & 119 & 29 & 57 & 228 & 458 & $<$ LOD & 1.07 & 0.73 & 4.52 \\
\hline mec $09-02 \mathrm{a}-2$ & Ignimbrite & 11R 707506 & 3305647 & Cataviña B.C. & 207 & 69 & 121 & 28 & 62 & 244 & 402 & $<$ LOD & 1.09 & 0.82 & 4.48 \\
\hline $\operatorname{mec} 09-02$ a-3 & Ignimbrite & 11R 707507 & 3305648 & Cataviña B.C. & 223 & 76 & 121 & 25 & 62 & 237 & 252 & $<$ LOD & 1.08 & 0.77 & 4.36 \\
\hline ep 07-1a-1 & Ignimbrite & 12R 517371 & 3165548 & Sierra Libre Son. & 306 & 40 & 252 & 34 & 192 & 198 & 336 & 314 & 1.13 & 0.58 & 2.96 \\
\hline ep 07-1a-2 & Ignimbrite & 12R 517372 & 3165549 & Sierra Libre Son. & 280 & 62 & 211 & 24 & 210 & 181 & 412 & 219 & 1.16 & 0.64 & 2.82 \\
\hline ep $07-1 a-3$ & Ignimbrite & 12R 517373 & 3165550 & Sierra Libre Son. & 380 & 59 & 223 & 34 & 190 & 233 & 485 & 273 & 1.29 & $<\mathrm{LOD}$ & 2.81 \\
\hline lps1 09-03-1 & Lava & 12R 503562 & 3162260 & Sierra Libre Son. & 483 & $<$ LOD & 214 & 27 & 84 & 168 & 220 & $<$ LOD & 1.1 & $<$ LOD & 4.74 \\
\hline lpsl 09-03-2 & Lava & 12R 503563 & 3162261 & Sierra Libre Son. & 493 & $<$ LOD & 221 & 24 & 107 & 211 & 268 & $<$ LOD & 1.15 & $<$ LOD & 4.66 \\
\hline lps1 09-03-3 & Lava & 12R 503564 & 3162262 & Sierra Libre Son. & 495 & $<$ LOD & 207 & 23 & 87 & 179 & 188 & $<$ LOD & 1.15 & $<\mathrm{LOD}$ & 4.53 \\
\hline
\end{tabular}

Table 2.- Trace element and major element concentrations from rhyolite slabs studied by a ED-XRF spectrometer. $<$ LOD, below limit of detection. Tabla 2.- Concentración de elementos traza y mayores en secciones de riolitas, mediante espectrómetro ED-FRX. $<$ LOD, por debajo del límite de detección. 

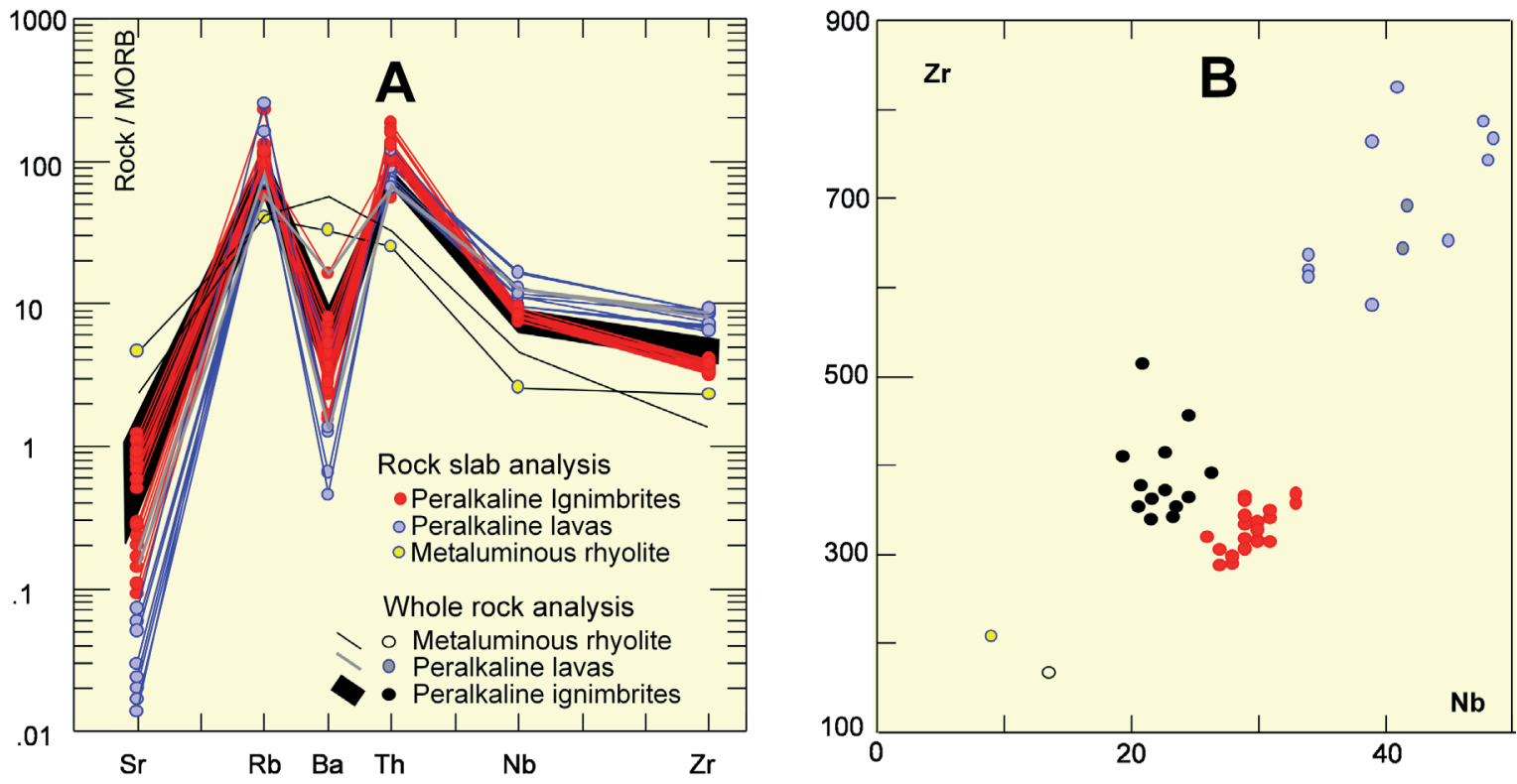

Fig. 3.- Diagrams illustrating the chemical identity of the samples analyzed: A, MORB normalized multi-element diagram of Pearce (1983); B, variation diagram of $\mathrm{Nb}$ vs Zr. See discussion in text. Values previously determined by analysis of whole-rock samples by ICP (Vidal-Solano, 2005; Vidal-Solano et al., 2005; Vidal-Solano et al., 2008a, 2008b; Olguin-Villa 2010) are included in these and subsequent diagrams to illustrate the good correlation values obtained by the rock slab analysis techniques.

Fig. 3.- Diagramas que ilustran la identidad química de las muestras analizadas: A, diagrama multielemental normalizado a MORB de Pearce (1983); B, diagrama de variación Zr vs Nb. Ver discusión en el texto. Valores previamente obtenidos por medio de análisis de roca total (Vidal-Solano, 2005; Vidal-Solano et al., 2005; Vidal-Solano et al., 2008a, 2008b; Olguin-Villa 2010) están incluidos aquí y en las figuras siguientes para mostrar la buena correlación de los valores obtenidos con las técnicas de análisis de las secciones de roca.

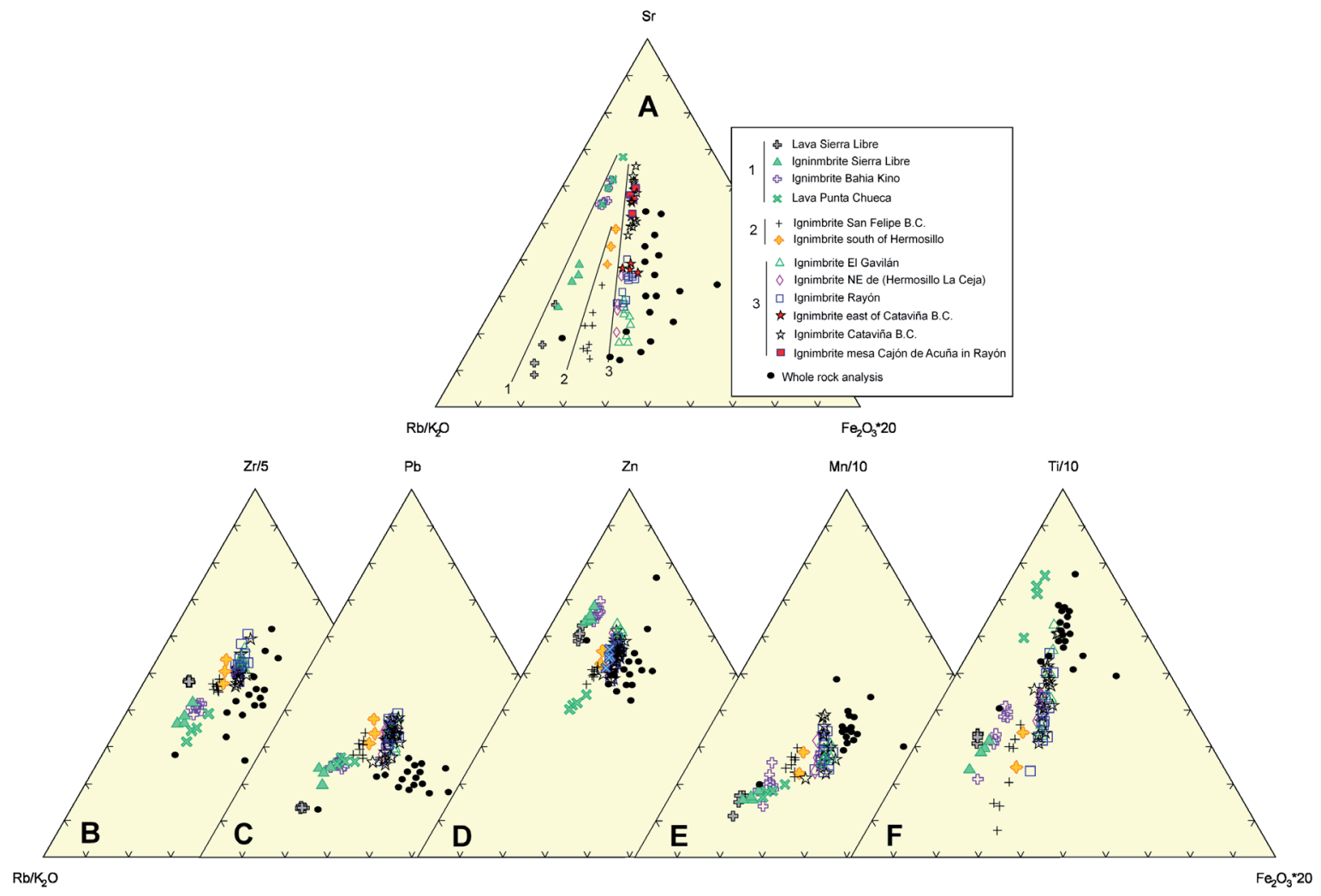

Fig. 4.- Ternary diagrams used in chemical discrimination and correlation of the ignimbrite deposits studied, built with the major and trace elements that are best represented in these rocks. The combination of these elements distinguishes three chemical groups probably derived from magma differentiation processes.

Fig. 4.- Diagramas ternarios utilizados en la discriminación química y la correlación entre los depósitos ignimbríticos estudiados, construidos con los elementos mayores y traza mejor representados en estas rocas. La combinación de estos elementos permite reconocer tres grupos químicos probablemente derivados de los procesos de diferenciación de los magmas. 
ized by pronounced peaks in $\mathrm{Rb}$ and $\mathrm{Th}$, moderate peaks in $\mathrm{Nb}$ and $\mathrm{Zr}$, and negative anomalies in $\mathrm{Ba}$ and $\mathrm{Sr}$, due to feldspar fractionation. The rhyolitic lavas present similar patterns, but more pronounced negative anomalies and a higher degree of enrichment in $\mathrm{Nb}$ and $\mathrm{Zr}$. The parallelism of the spectra of ignimbrites and rhyolites supports a common source for all the peralkaline samples. It is also noted that it is possible to differentiate between the concentrations of the rhyolitic ignimbrite and lava flow samples. The ratio between concentrations of elements in the peralkaline rhyolites, which is visibly different from one of the metaluminous rhyolites analyzed, imparts in these lavas a unique geochemical identity (Fig. 3A).

Concentrations of incompatible and immobile elements $\mathrm{Nb}, \mathrm{Zr}$ and Th, called HFSE (High Field Strength Elements), are highly variable for the rhyolitic samples, while the points representing the ignimbrite samples plot in a limited area (Fig. 3B) illustrating a good correlation between all the studied ignimbrite outcrops.

For a better chemical discrimination we plot on ternary diagrams trace and major elements with sufficient abundance in these rocks (Fig. 4). On the $\mathrm{Rb} / \mathrm{K} 2 \mathrm{O}-\mathrm{Sr}$ Fe2O3 diagram (Fig. 4A), we can discriminate 3 different trends: a) one corresponding to the ignimbrite and lavas from Sierra Libre, Bahía de Kino and Punta Chueca; b) a second trend corresponding to samples from Hermosillo and those from San Felipe in Baja California and finally, c) a third trend that brings together most of the Sonora and Baja California outcrops. Each group shows a strong variation in $\mathrm{Sr}$ contents, which can be attributed to different percentages of alkali feldspar fractionation. The distal ignimbritic deposits from Cataviña (Baja California) and Rayón (Sonora), which are separated by more than 500 $\mathrm{km}$, plot in the same trend. Most particularly, the vitrophyre samples from Mesa Cajón de Acuña in Rayon (Sonora) fall in the same group (with higher Sr content) as the Cataviña samples (Olguin-Villa, 2010; Olguin-Villa et al., 2010, Gomez-Valencia and Vidal-Solano, 2010; Olguin-Villa et al., 2013).

\section{Discussion}

\subsection{Reliability of the approach}

Several factors can influence the reproducibility of the chemical signature of the studied samples: a) the percentage of glass and phenocrysts, b) the presence of xenocrysts and, c) the presence of xenoliths or lithic clasts. Xenoliths can be easily detected because a petrographic study is conducted at the same time on the analyzed samples. Some porphyritic samples can contain phenocrysts that come from a different liquid; these xenocrysts are not in equilibrium with the peralkaline liquid and therefore introduce discrepancies in the data. This factor is however negligible as all the vitreous samples studied in this work have more than $85 \%$ glass.

The greatest difference in composition was observed in rock slabs of Cataviña pyroclastic outcrops in Baja California (Table 1 and 2, group 3 in Fig. 4A), where the same ignimbrite unit shows variations in xenocryst contents ranging from 5 to $15 \%$. These crystals such as plagioclase, biotite, hornblende and orthopyroxene are easily identified as phenocrysts because they do not correspond to the mineral phase association observed in peralkaline magmatism. In accord with previous analysis, results obtained by this approach on obsidians at the base of some peralkaline rhyolitic lava flows show also low $\mathrm{Sr}$ values that can be related to different percentages of alkali feldspar fractionation. Excellent geochemical correlations as well as characterization of major and trace element variations in the peralkaline pyroclastic deposits were obtained by analyzing flat surfaces of rock slabs, giving evidence of the reliability of this approach.

\subsection{Regional implications}

The variations of the concentrations in $\mathrm{Zr}, \mathrm{Pb}, \mathrm{Zn}, \mathrm{Mn}$ and Ti (Figs. 4B, C, D, E and F) established that ignimbrite samples range from low values (except for $\mathrm{Zn}$ which has a reverse behavior) in thicker deposits such as those of Sierra Libre or Kino Bay, up to higher values in the distal deposits of reduced thickness that correspond to the boundary of the ignimbrite exposures, such as those of the Rayón and Cataviña areas (Fig. 1). During a sustained large-volume eruption, successive asymmetric lobes of an ignimbrite may develop in different locations (Branney and Kokelaar, 2002). Sequentially developed regional lobes may be difficult to distinguish within an extensive ignimbrite sheet, but can be revealed by detailed fieldwork coupled with petrographic analysis in cases where the composition of the eruption or included lithic clasts changed with time (e.g. Bishop Tuff; Wilson \& Hildreth 1997). Such a distribution of the thickness and chemical variations suggest that all of these peralkaline pyroclastic flow deposits in NW Mexico are directly associated and related to a unique and large event (mega-eruption). Oskin (2002) and Oskin and Stock (2003) proposed that the vent area of the Tuff of San Felipe was located at Punta Chueca, north of Kino Bay, because of the unusual characteristics of the base of the deposit. In that location the ignimbrite does not have a defined base, with a vitrophyre, but rather appears to continue downward into fluidal rhyolites, suggesting that the ignimbrite corresponds to an intracaldera facies rather than an outflow 
sheet. Although the ignimbrite does have a basal vitrophyre in one part of the Sierra Libre, we consider Sierra Libre as a more appropriate vent location, because great volumes of peralkaline rhyolite lavas have been found there in clear stratigraphic relationship with peralkaline ignimbrites (Barrera-Guerrero and Vidal-Solano, 2010; Barrera-Guerrero, 2012). The chemical variations and the geographic distribution of ignimbrite deposits may suggest that they are related to a zoned magma reservoir. The detailed relationships of the lavas with the ignimbrite at Punta Chueca and at Sierra Libre, and the interpretation of the basal relationships of the ignimbrite in the vicinity of these two locations, remain to be determined. Nevertheless, we propose that both of these regions were originally adjacent and part of the same vent system or at least the same volcanic field. Our proposal is based on stratigraphic and geochemical correlation of the peralkaline units in the volcanic sequence of both regions.

An excellent correlation is observed for group 1 ignimbrites (Fig. 4) that comprise the Sierra Libre and Kino Bay outcrops, which are separated by more than $100 \mathrm{~km}$. Recently, Bennett (2009) has proposed the existence of a rapid Late Miocene west-northwest-directed transtensional displacement across the Kino Bay area, which could represent some of the Pacific-North America (PAC-NAM) plate boundary deformation. However, this dextral shear zone localized within the Kino Bay area is not sufficient to successfully accommodate the $\sim 150 \mathrm{~km}$ missing after the paleo-tectonic restoration of the post6.1 Ma PAC-NAM plate boundary position (Oskin et al., 2001). On the basis of the chemical correlations, and our existing understanding of the basal characteristics of the ignimbrite, we propose the existence of dextral faults between Kino Bay and Sierra Libre, which may have displaced crustal blocks during a period of strong tectonic activity (12-6 Ma) related to a proto-Gulf transtensional episode (Fig. 1). The outcrop distribution of the Sonora samples suggests a displacement of at least $100 \mathrm{~km}$ that affected the ignimbrites of coastal Sonora, during a Late Miocene transtensional event prior to the opening of the Gulf of California. This major structure would now be buried by the large amount of sediment that has been deposited in recent deltas on the Sonora coast.

\section{Conclusions}

The combination of analytical methods applied in this work lead to several main conclusions [i] WD-XRF and ED-XRF analyses of surfaces of rock slabs are an excellent complement to the petrological characterization of vitreous samples, particularly those from pyroclastic density current deposits in which the lithic influence on whole rock analysis cannot be ignored. [ii] This kind of analysis allows us to determine the peralkaline character of the Middle Miocene anorogenic rhyolites (ignimbrites and lava flows) and to discriminate them from the metaluminous rhyolites. [iii] By applying this method to the regional ignimbrites, we are able to correlate localities separated by hundreds of kilometers, supporting the hypothesis that all the studied ignimbrite vitrophyres stem from a unique mega-eruption. [iv] Our regional correlations suggest displacement (up to more than $100 \mathrm{Km}$ ) between some of the thickest ignimbrite deposits, which we attribute to now-buried dextral faults related to the protoGulf of California opening during Middle-Late Miocene time.

\section{Acknowledgements}

This work was supported by a Research Grant \#061198 from Consejo Nacional de Ciencia y Tecnología (CONACYT) to Jesús Roberto Vidal Solano. Thanks to Sheila A. Corrales (Departamento de Geología de la Universidad de Sonora) for thin-section and rock slab preparation. J. Stock's participation was supported by the US National Science Foundation grant EAR-0911761. The authors wish to thank reviewers Dr. Takeshi Kuritani and Dr. Manoj. K. Pandit for valuable comments that have enriched this work. Likewise, the detailed and careful comments of Dr. Alain Demant are greatly appreciated.

\section{References}

Barrera-Guerrero, S. (2012): Contribución al estudio del evento volcánico hiperalcalino del Mioceno Medio en el NW de México: Petrología de la Sierra Libre, Sonora. (M.S. thesis): Departamento de Geología, Universidad de Sonora, 114pp.

Barrera-Guerrero, S., Vidal-Solano J.R. (2010): Reconocimiento del volcanismo hiperalcalino de edad Mioceno Medio en el NW de México: Un registro completo en La Sierra Libre, Sonora, México. $X X$ Congreso Nacional de Geoquímica, Temixco, Morelos, México. Actas INAGEQ, 16, 229-234.

Bennett, S. (2009): Transtensional Rifting in the Late Proto-Gulf of California Near Bahía Kino, Sonora, México. (M.S. thesis): University of North Carolina at Chapel Hill, $122 \mathrm{p}$.

Branney, M. J., Kokelaar, P. (2002): Pyroclastic Density Currents and the Sedimentation of Ignimbrites. Geological Society, London, Memoirs, 27.

Gomez-Valencia A. M., Vidal-Solano J.R. (2010): Los depósitos ignimbríticos anorogénicos en Rayón, Sonora: Correlación con el volcanismo hiperalcalino del Mioceno Medio en el NW de México. $X X$ Congreso Nacional de Geoquímica, Temixco, Morelos, México: Actas INAGEQ, 16, 241-246.

Hernández-Méndez, G.L., Stock, J., Vidal-Solano, J., Paz-Moreno, F.A. (2008): Paleomagnetic constraints on the extent of the Miocene Tuff of San Felipe / Tuff of Hermosillo, Sonora, Mexico. Abstract 197-2, Geological Society of America Abstracts with Programs 40(6), 264.

Lozano, R., Bernal, J.P. (2005): Assessment of a new set of geochemical reference materials for XRF major and trace element analysis. Revista 
Mexicana de Ciencias Geológicas, 22, 329-344.

Lozano, R., Vidal-Solano, J.R., Zamora, O., Mendoza-Córdova, A. (2010): Determinación de elementos traza por FRX en vidrios volcánicos mediante el análisis de "testigos" de láminas delgadas: Identidad geoquímica de las riolitas hiperalcalinas del NW de México. $X X$ Congreso Nacional de Geoquímica, Temixco, Morelos, México: Actas INAGEQ, 16, 170-175.

Olguín-Villa, A.E. (2010): Estudio fisico y químico del volcanismo hiperalcalino en la región de Cataviña, Baja California. Tesis de Licenciatura, Universidad de Sonora, $84 \mathrm{p}$.

Olguín-Villa, A.E. (2013): Establecimiento de la estratigrafía magnética del evento volcánico hiperalcalino del Mioceno Medio en la Sierra Libre, Sonora, México. (M.S. thesis): Departamento de Geología, Universidad de Sonora, México, 76pp.

Olguín-Villa, A.E., Vidal-Solano, J.R., Stock, J.M. (2010): Geoquímica del volcanismo félsico de edad Mioceno medio en la región de Cataviña: significado del volcanismo hiperalcalino en Baja California, México. XX Congreso Nacional de Geoquímica, Temixco, Morelos, México: Actas INAGEQ, 16, 247-252.

Olguín-Villa, A.E., Vidal-Solano, J.R., Stock J.M. (2013): Petrografía, geoquímica, petro-fábrica y paleomagnetismo de la Toba de San Felipe en la región de Cataviñá, Baja California, México. Revista Mexicana de Ciencias Geológicas, 30, in press.

Oskin, M.E. (2002): Tectonic evolution of the northern Gulf of California, México, deduced from conjugate rifted margins of the upper Delfín Basin. PhD thesis California Institute of Technology, Pasadena, $487 \mathrm{p}$.

Oskin, M, Stock, J., Martin-Barajas, A. (2001): Rapid localization of Pacific-North America plate motion in the Gulf of California. Geology, 29, 459-462. doi:10.1130/0091-7613(2001)029<0459:RLOPNA $>2.0 . \mathrm{CO} ; 2$

Oskin M.E., Stock J.M., (2003): Cenozoic volcanism and tectonics of the continental margins of the Upper Delfin basin, northeastern Baja California and western Sonora. In S.E. Johnson, S.R. Patterson, J.M. Fletcher, G.H. Girty, D.L. Kimbrough \& A. Martín-Barajas (Eds.), Tectonic evolution of northwestern México and the southwestern USA. Geol. Soc. Am. Spec. Paper 374, 421-438. doi:10.1130/0-81372374-4.421

Pearce, J.A. (1983): Role of the sub-continental lithosphere in magma genesis at active continental margins, In Hawkesworth, C. J. and Norry, M. J. eds., Continental basalts and mantle Xenoliths, 230-250, Shiva, Nantwich, Cheshire, U.K.

Stock, J.M., Lewis, C.J., Nagy, E.A. (1999): The Tuff of San Felipe: an extensive Middle Miocene pyroclastic flow deposit in Baja California, Mexico, Journal of Volcanology and Geothermal Research, 93, 53-74. doi:10.1016/S0377-0273(99)00079-7

Stock, J.M., Martín-Barajas, J.A., Chapman, A., López-Martínez, M. (2008): Net Slip across the Ballenas Transform Fault Measured from Offset Ignimbrite deposits. EOS Trans. American Geophysical Union, Fall Meeting Supplement, 89(53), abstract \#T11A-1853.

Vidal-Solano, J.R. (2005): Le volcanisme hyperalcalin d'âge Miocène Moyen du Nord-Ouest du Mexique (Sonora): Minéralogie, Géochimie, cadre géodynamique. Ph.D. Thesis, Université Paul Cézanne, 256p.

Vidal-Solano, J.R. (2012): Estudio petrológico de los Paleo-Volcanes hiperalcalinos de Sonora, México. Epistemus, 13, 21-26.

Vidal-Solano, J.R., Paz-Moreno, F.A., Iriondo, A., Demant, A., Cochemé, J.J. (2005): Middle Miocene peralkaline ignimbrites in the Hermosillo region (Sonora, Mexico): Geodynamic implications. $C$. R. Geoscience, 337, 1393-1582. doi:10.1016/j.crte.2005.08.007

Vidal-Solano, J.R., Paz-Moreno, F.A., Demant, A., López-Martínez, M. (2007): Ignimbritas hiperalcalinas del Mioceno medio en Sonora Central; Revaluación de la estratigrafía y significado del volcanismo Terciario. Revista Mexicana de Ciencias Geológicas, 24, 47-67.

Vidal-Solano, J.R., Stock, J.M., Iriondo, A., Paz-Moreno, F.A. (2008a): Las ignimbritas hiperalcalinas del NW de México: Una mega erupción durante el Mioceno medio?. Reunion Anual de la Union Geofísica Mexicana, Pto. Vallarta, Jalisco, México. Geos, 28, p. 218.

Vidal-Solano, J.R., Demant A., Paz-Moreno, F.A., Lapierre, H., OrtegaRivera M.A., Lee, J.K.W. (2008b): Insights into the tectonomagmatic evolution of NW Mexico: Geochronology and geochemistry of the Miocene volcanic rocks from the Pinacate area, Sonora. Geological Society of America Bulletin, 120, 691-708. doi:10.1130/B26053.1

Vidal-Solano J.R. y Meza-Figueroa D.M. (2009): En búsqueda de una correlación geoquímica para las ignimbritas hiperalcalinas del Mioceno Medio en el NW de México: Avances en el análisis de láminas delgadas con ICP-AES y un sistema de ablación laser acoplado. XIX Congreso Nacional de Geoquímica, Ensenada, Baja California, México. Actas INAGEQ, 15 (1), 50-51.

Wilson, C. J. N., Hildreth, W. (1997). The Bishop Tuff: new insights from eruptive stratigraphy. Journal of Geology, 105, 407-439. doi:10.1086/515937

Zamora Martínez, O., Martín Romero, F., Lozano Santa Cruz, R. (2008): Evaluación del desempeño de un analizador portátil de Fluorescencia de Rayos X en la determinación de la composición elemental de residuos mineros. Reunion Anual de la Union Geofísica Mexicana, Pto. Vallarta, Jalisco, México. Geos, 28 (2), p-161. 